\title{
A financial analysis of operating room charges for robot-assisted gynaecologic surgery: Efficiency strategies in the operating room for reducing the costs
}

\author{
Burak Zeybek ${ }^{1}$, Tufan Öge'2, Cemil Hakan KIlıç3, Mostafa A. Borahay, Gökhan Sami Kılıç \\ 'Department of Obstetrics and Gynecology, Ege University Faculty of Medicine, Izmir, Turkey \\ ${ }^{2}$ Department of Obstetrics and Gynecology, Eskişehir Osmangazi University Faculty of Medicine, Eskişehir, Turkey \\ ${ }^{3}$ Counseler, Istanbul Chamber of Commerce, Istanbul, Turkey \\ ${ }^{4}$ Department of Obstetrics and Gynecology, The University of Texas Medical Branch at Galveston, Galveston, USA
}

\section{Abstract}

Objective: To analyse the steps taking place in the operating room (OR) before the console time starts in robot-assisted gynaecologic surgery and to identify potential ways to decrease non-operative time in the OR.

Material and Methods: Thirteen consecutive robotic cases for benign gynaecologic disease at the Department of Obstetrics and Gynecology at University of Texas Medical Branch (UTMB) were retrospectively reviewed. The collected data included the specific terms 'Anaesthesia Done' (step 1), 'Drape Done' (step 2), and 'Trocar In' (step 3), all of which refer to the time before the actual surgery began and OR charges were evaluated as level 3,4 , and 5 for open abdominal/vaginal hysterectomy, laparoscopic hysterectomy, and robot-assisted hysterectomy, respectively.

Results: The cost of the OR for 0-30 minutes and each additional 30 minutes were $\$ 3,693$ and $\$ 1,488, \$ 4,961$ and $\$ 2,426$, $\$ 5,513$ and $\$ 2,756$ in level 3,4 , and 5 surgeries, respectively. The median time for step 1 was $12.1 \mathrm{~min}$ (5.25-23.3), for step 2 was 19 (4.59-44) min, and for step 3 was 25.3 (16.45-45) min. The total median time until the actual operation began was $54.58 \mathrm{~min}(40-100)$. The total cost was $\$ 6948.7$ when the charge was calculated according to level 4 and $\$ 7771.1$ when the charge was calculated according to level 5 .

Conclusion: Robot-assisted surgery is already 'cost-expensive' in the preparation stage of a surgical procedure during anaesthesia induction and draping of the patient because of charging levels. Every effort should be made to shorten the time and reduce the number of instruments used without compromising care. (J Turk Ger Gynecol Assoc 2014; 15: 25-9)

Key words: Cost analysis, fees, gynaecology operating rooms, robot-assisted surgery

Received: 24 October, 2013

Accepted: 05 November, 2013

\section{Introduction}

The da Vinci Surgical System (Intuitive Surgical, Sunnyvale, CA, USA) has been the subject of debate with regard to high costs since its first approval by the U.S. Food and Drug Administration in 2001 for general surgery. This system is the only approved robotic surgical unit and has fixed costs, with prices for each unit ranging from $\$ 1$ million to $\$ 2.5$ million, as well as annual maintenance costs and the costs of additional consumables (1). To date, although procedurebased analyses have demonstrated higher costs for robotic surgery compared with conventional laparoscopic surgery and abdominal/vaginal surgery, robotic surgery has still been increasingly used, and more than 1,900 robotic surgical systems have been installed worldwide (2-5). Moreover, robotassisted surgery in gynaecology have become an important area in residency training programmes (6).
Minimally invasive surgery (MIS) is rapidly gaining popularity in the field of gynaecology and there has been a shift towards MIS in the last 10 years in US (7). This shift was dramatically increased after the approval of robotic-assisted surgery in 2005 and is still being used for a variety of applications including hysterectomy, sacrocolpopexy, tubal reanastomosis, and radical oncological operations $(2-4,8)$. Initially, the number of opponents of robotic surgery was much higher than the number of supporters, and there was a clear trend towards not using the robot because of its high costs and similar clinical benefits compared with laparoscopy. However, the clear advantages over traditional laparoscopy, including threedimensional imaging, tremor filtration, augmented dexterity, and surgeon comfort, have led physicians to investigate approaches to reduce costs without compromising care (9). The absolute costs of procedures in the American health care system are calculated according to operative time, surgical 
complications, length of stay, and other probable cost drivers compared to hospital estimates and individually negotiated reimbursement agreements. Operating room (OR) charges are one of the most important factors affecting the total cost of procedures, and the two main factors that affect the OR cost are the time spent in the OR and the OR charging levels. Currently, medical data focuses on the actual surgery time, console time, and total operating-room time $(4,7,10)$. Less emphasis is placed on the time frame before the console time starts.

In this study, we analysed the steps taking place in the operating room before the console time starts and discuss potential ways to decrease non-operative time in the OR.

\section{Material and Methods}

Thirteen consecutive robotic cases for benign gynaecologic disease at the Department of Obstetrics and Gynecology at University of Texas Medical Branch (UTMB) were retrospectively reviewed. Institutional Review Committee approval was obtained. Patient data were arranged in chronological order. The collected data included the specific terms 'Anaesthesia Done', 'Drape Done', and 'Trocars Placed In', all of which refer to the time before the actual surgery began. In the presence of faculty members from the anaesthesia and gynaecology departments as well as the circulating nurse, the patient is physically wheeled into the operating room. Safety checks were completed before the induction of anaesthesia was initiated. A second intravenous line (IV) opened after intubation was securely completed. 'Anaesthesia Done' was defined as the time period that started from the patient being wheeled into the operating room and ran until the second IV line was opened; this included time out, intubation, and secondary intravenous access. 'Drape Done' was defined as the time period that included patient positioning, preparation, and draping. 'Trocars Placed In' was defined as the placement of the uterine manipulator and when all trocars had been introduced into the abdominal cavity. The docking time and the operating time were not included in the study. This also helped to translate the data obtained from this study to laparoscopic cases. The time during 'Anaesthesia Done' was defined as 'step 1', that during 'Drape Done' was defined as 'step 2' and 'Trocars Placed In' was defined as 'step 3 '. Because the amount of time lapsed until the actual operation begins is identical in both robotic and conventional laparoscopic surgery, we did not create a study group for patients who underwent laparoscopic surgery.

\section{Surgery Levels}

According to UTMB Charge Description Master (CDM) standardisation, a formula was devised for an organisation-wide standard for the number of surgery levels and the criteria for categorising surgical procedures into a specific surgery level based on five drivers (Table 1). These drivers are elements in a surgical procedure that allow for differentiation based on the complexity of the procedure.

The following information presents the drivers that guided the surgical team in determining the number of surgery levels in the protocol along with the characteristics of each level:
A. Equipment: Degree of specialised equipment required

B. Instruments: Number of trays/kits

C. Setup time: Required preparation and teardown of room

D. Staff: Number of staff (defined as hospital employees)

E. Supplies: Routine in nature (non-billable)

Charges for OR services generally depend on the complexity of the particular operation. There is an initial charge, followed by an additional charge for every 30 minutes (Table 2). Robotic surgery charges are based on level 5, whereas laparoscopic surgery charges are based on level 4, and open abdominal hysterectomy and vaginal hysterectomy charges are based on level 3. These financial data were obtained from the Hospital Financial Management and Physician Billing Departments. The cost of the OR for 0-30 minutes and each additional 30 minutes was evaluated.

The da Vinci Surgical System (Intuitive Surgical, Sunnyvale, CA, USA) was used for the robotic surgical procedures, whereas traditional laparoscopic instruments were utilised for laparoscopic surgery.

\section{Statistical Analysis}

Descriptive statistics were calculated, and the median and range were examined. Statistical analyses were performed using the Statistical Package for Social Science for Windows Version 20.0 (SPSS Inc., Chicago, IL, USA).

\section{Results}

The results are presented as median and ranges. The median time for step 1 was 12.1 min (5.25-23.3), for step 2 was 19 (4.59-44) min, and for step 3 was 25.3 (16.45-45) min (Figure 1). The total median time until the actual operation began was $54.58 \mathrm{~min}$ (40-100). The cost of OR for 0-30 minutes and each additional 30 minutes were $\$ 3,693$ and $\$ 1,488, \$ 4,961$ and $\$ 2,426$, and $\$ 5,513$ and $\$ 2,756$ in level 3,4 , and 5 surgeries, respectively (Table 2).

If the charge was calculated according to level 4 , then the costs of step 1, step 2 and step 3 were $\$ 2000.9$, $\$ 3049$ and $\$ 2045.9$, respectively. If the charge was calculated according to level 5, then the costs of step 1, step 2 and step 3 were $\$ 2223.6$, $\$ 3390.5$ and $\$ 2296.6$, respectively.

In a total median time of 54.58 minutes, the total costs were $\$ 6948.7$ and $\$ 7771.1$ when the charge was calculated according to level 4 and level 5, respectively (Table 3 ). The absolute difference between the robotic and laparoscopic cases was $\$ 822$.

\section{Discussion}

To the best of our knowledge, this study is the first report focusing primarily on performing a thorough evaluation of the time period prior to the actual beginning of the operation. This time period is identical for both laparoscopic and robotic techniques. Developing new approaches to decrease the time frame before the actual surgery started would be cost effective for both robotic and laparoscopic techniques.

Efficiency, in dictionaries, describes the extent to which time, effort or cost is well used for the intended task or purpose, and 
Table 1. UTMB CDM standardised surgery levels

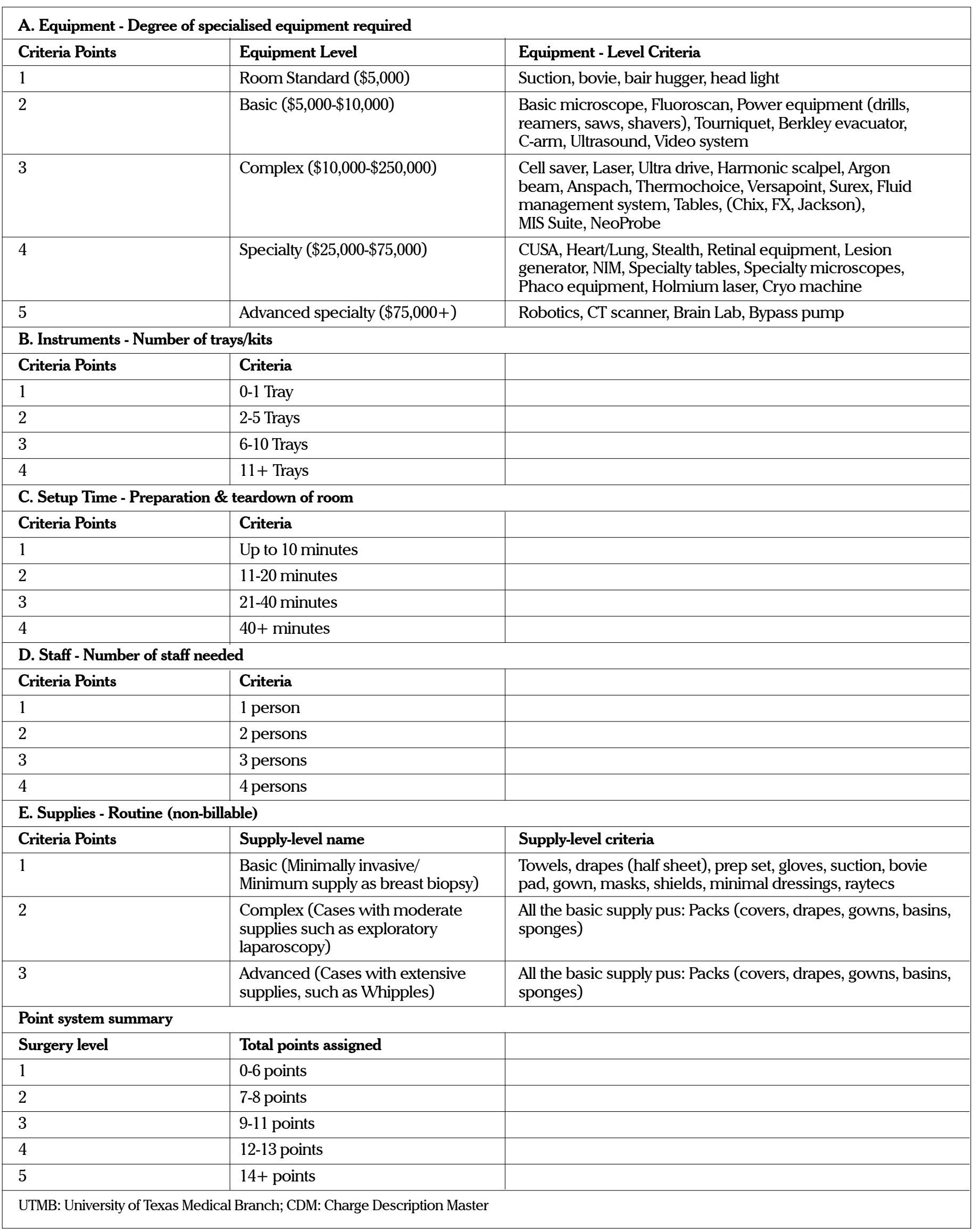


Table 2. Operating room charges of abdominal/vaginal, laparoscopic and robotic

\begin{tabular}{|l|c|c|}
\hline Complexity level & Initial 30 $\mathbf{~ m i n}$ (\$) & $\begin{array}{c}\text { Each additional per } \\
\mathbf{3 0} \text { min (\$) }\end{array}$ \\
\hline 3 & 3.693 & 1.488 \\
\hline 4 & 4.961 & 2.426 \\
\hline 5 & 5.513 & 2.756 \\
\hline $\begin{array}{l}\text { Level 3: Abdominal/Vaginal } \\
\text { Level 4: Laparoscopy } \\
\text { Level 5: Robot }\end{array}$ \\
\hline
\end{tabular}

Table 3. Costs of each step according to complexity levels of the procedures before the surgery begins

\begin{tabular}{|l|c|c|c|}
\hline Cost (\$) & $\begin{array}{c}\text { Level 3 } \\
\text { (Abdominal/ } \\
\text { Vaginal) }\end{array}$ & $\begin{array}{c}\text { Level 4 } \\
\text { (Laparoscopy) }\end{array}$ & $\begin{array}{c}\text { Level 5 } \\
\text { (Robot) }\end{array}$ \\
\hline Step 1 & 1489.5 & 2000.9 & 2223.6 \\
\hline Step 2 & 2258 & 3049 & 3390.5 \\
\hline Step 3 & 1254.9 & 2045.9 & 2296.6 \\
\hline Total & 4912.2 & 6948.7 & 7771.1 \\
\hline
\end{tabular}

efficiency could be implemented in the OR to eliminate wasteful activities. To maintain efficiency in the OR, first, a dedicated room for robotic surgery is needed. The OR should be large (approximately $60 \mathrm{~m}^{2}$ ) and should include back-up materials in case of potential equipment malfunctions (9). Once the physical plant of the OR is setup appropriately, it is of vital importance to create a cooperative team for the efficiency of the program. Fagin published a model for the efficient utilisation of the OR and argued that a team including five dedicated people (two scrub techs, one circulator, one first assistant, one operating room attendant) is sufficient to perform parallel tasking (task overlap) in the robotic OR and described 'task overlap' as performing tasks in parallel rather than in a consecutive series (11). To succeed in this efficiency model, individual team member roles must be defined, standardised and the task overlap should be carried out effectively. In general, the circulator is responsible for the overall flow of the room, scrub techs should be proficient in organising procedure-based surgical instruments and the first assistant (or resident/fellow), who is the bedside colleague of the surgeon, should be proficient in manipulating tissue and managing instruments. Teaching and training the team are very important because any inefficient behaviour increases the amount of time required to perform the steps, leading to higher costs for the procedures. However, all of the people involved in the robotic program have their own learning curve; therefore, no more than one person should be trained at a time, as increasing the number of trainees will also increase the length of the operation. Additionally, each team member, including the surgeon, must be actively involved in the parallel tasking. For example, when the patient enters the room, the surgeon (or resident/fellow) and the circulator should position and prep the patient while the scrub tech is focused on preparing the table and draping the robot.
Our study shows that in a median time of 55 minutes before the actual operation begins, the use of the robotic system results in an additional $\$ 2858.9$ in charges when compared with open abdominal hysterectomy or vaginal hysterectomy, indicating that robotic surgery has already become dramatically more expensive at the beginning of the procedure. If evaluated step-bystep, there was an additional cost of $\$ 734$ with regard to step 1, $\$ 1132$ with regard to step 2, and \$1042 with regard to step 3 . Although step 3 was the longest, its costs were lower than those of step 2 because the OR charges differ after the first 30 minutes. To shorten the time period and decrease the cost for step 1 in an efficient way, time-out can be finished before the patient enters the room or after the patient has been intubated and intravenous lines can be accessed before the patient enters the OR. The key point for shortening the time for step 2, which is the most expensive step, is that 'parallel tasking' must be carried out very effectively in this period. The circulator should focus on positioning the patient, the scrub tech should make the table ready, and the resident/fellow should prep and drape the patient all at the same time. Once the patient is draped, the surgeon should be ready with abdominal access to make the initial incision, insufflate the abdomen and finally place the trocars. At this stage, the team should know the order of the surgeon's tasks very well, as the circulator can connect the gas prior to insufflation and the scrub tech can make the instruments ready according to the task order. This anticipation will contribute to shortening the time period and decreasing the cost for step 3 . All of these methods can be applied to both laparoscopic and robotic procedures, thus leading to more comparable costs with abdominal/vaginal procedures. One other reason causing these high costs is likely related to the increased equipment levels and an increased number of instruments that need to be prepared for robotic surgery, leading to high charge levels, although some instruments are unnecessary. Moreover, the term 'robot' also adds to the complexity of the name of the surgery, thereby contributing to the high charge levels.

Our study also has certain limitations. The study is limited by its retrospective nature with inherent selection bias, and the data collection itself is subject to measurement bias as a result of errors in data gathering.

In conclusion, robotic surgery is already 'cost-expensive' in the preparation stage of a surgical procedure during anaesthesia induction and draping of the patient because of the charging levels. Every effort should be made to shorten the time and reduce the number of instruments without compromising care.

Ethics Committee Approval: Ethics committee approval was received for this study (IRB11-340).

Informed Consent: Written informed consent was not taken because this study was not involved direct patient contact.

Peer-review: Externally peer-reviewed.

Author contributions: Concept - G.S.K., B.Z.; Design - C.H.K., T.Ö.; Supervision - G.S.K.; Resource - G.S.K.; Materials - C.H.K., B.Z.; Data Collection\&/or Processing - G.S.K., M.A.B.; Analysis\&/ or Interpretation - C.H.K.; Literature Search - T.Ö.; Writing G.S.K.; Critical Reviews - G.S.K., M.A.B. 
Conflict of Interest: No conflict of interest was declared by the authors.

Financial Disclosure: The authors declared that this study has received no financial support.

\section{References}

1. Barbash GI, Glied SA. New technology and health care costs--the case of robot-assisted surgery. N Engl J Med 2010; 363: 701-4. [CrossRef]

2. Pasic RP, Rizzo JA, Fang H, Ross S, Moore M, Gunnarsson C. Comparing robot-assisted with conventional laparoscopic hysterectomy: impact on cost and clinical outcomes. J Minim Invasive Gynecol 2010; 17: 730-8. [CrossRef]

3. Paraiso MF, Jelovsek JE, Frick A, Chen CC, Barber MD. Laparoscopic compared with robotic sacrocolpopexy for vaginal prolapse: a randomized controlled trial. Obstet Gynecol 2011; 118: 1005-13. [CrossRef]

4. Wright KN, Jonsdottir GM, Jorgensen S, Shah N, Einarsson JI. Costs and outcomes of abdominal, vaginal, laparoscopic and robotic hysterectomies. JSLS 2012; 16: 519-24. [CrossRef]

5. Kilic GS, Moore G, Elbatanony A, Radecki C, Phelps JY, Borahay MA. Comparison of Perioperative Outcomes of Total Laparoscopic and Robotically Assisted Hysterectomy for Benign Pathology during Introduction of a Robotic Program. Obstet Gynecol Int 2011; 2011: 683703. [CrossRef]

6. Kilic GS, Walsh TM, Borahay M, Zeybek B, Wen M, Breitkopf D. Effect of residents' previous laparoscopic surgery experience on initial robotic suturing experience. ISRN Obstet Gynecol 2012; 2012: 569456. [CrossRef]

7. Wright JD, Ananth CV, Lewin SN, Burke WM, Lu YS, Neugut AI, et al. Robotically assisted vs laparoscopic hysterectomy among women with benign gynecologic disease. JAMA 2013; 309: 689-98. [CrossRef]

8. Rodgers AK, Goldberg JM, Hammel JP, Falcone T. Tubal anastomosis by robotic compared with outpatient minilaparotomy. Obstet Gynecol 2007; 109: 1375-80.[CrossRef]

9. Visco AG, Advincula AP. Robotic gynecologic surgery. Obstet Gynecol 2008; 112: 1369-84. [CrossRef]

10. Behera MA, Likes CE, 3rd, Judd JP, Barnett JC, Havrilesky LJ, $\mathrm{Wu} \mathrm{JM}$. Cost analysis of abdominal, laparoscopic, and roboticassisted myomectomies. J Minim Invasive Gynecol 2012; 19: 52-7. [CrossRef]

11. Fagin R. Achieving Efficiency in the Operating Room: Step by Step. In: Hemal AK, Menon M, editors. Robotics in Genitourinary Surgery. New York, USA: Springer; 2011.p.51-62. [CrossRef] 